\title{
Prognostic interaction between bone marrow morphology and SF3B1 and ASXL1 mutations in myelodysplastic syndromes with ring sideroblasts
}

\author{
Abhishek A. Mangaonkar', Terra L. Lasho' ${ }^{1}$, Christy M. Finke', Naseema Gangat ${ }^{1}$, Aref Al-Kali $\mathbb{D}^{1}$, Michelle A. Elliott ${ }^{1}$, \\ Kebede H. Begna ${ }^{1}$, Hassan Alkhateeb', Alexandra P. Wolanskyj-Spinner ${ }^{1}$, Curtis A. Hanson², Rhett P. Ketterling ${ }^{2}$, \\ William J. Hogan (1)', Animesh Pardanani ${ }^{1}$, Mark R. Litzow' ${ }^{1}$, Ayalew Tefferi' and Mrinal M. Patnaik (1)
}

The 2016 iteration of the World Health Organization (WHO) classification of myeloid neoplasms includes myelodysplastic syndrome with ring sideroblasts (MDSRS) as a unique sub-type of MDS. Presence of dysplasia in single or multiple cell lineages with at least $15 \%$ ring sideroblasts (RS), or as few as 5\% RS in the presence of SF3B1 mutations, further subcategorizes "MDS-RS" into: MDS-RS with single lineage dysplasia (SLD) and MDS-RS with multi-lineage dysplasia (MLD) ${ }^{1}$. In the prior 2008 WHO classification, MDS-RS-SLD was termed as refractory anemia with ringed sideroblasts and MDS-RSMLD was included under refractory cytopenia with multilineage dysplasia, a category that also incorporated MDS patients with MLD without $\mathrm{RS}^{2}$. These changes in classification were prompted on the basis of morphological distinctions and their impact on prognosis. In addition, the strong phenotypic correlation of SF3B1 mutations with bone marrow (BM) $\mathrm{RS}^{3}$ and the prognostic irrelevance of BM RS percentage ${ }^{4}$, resulted in the inclusion of these mutations in the classification schema. The advent of next generation sequencing has established the molecular landscape in MDS, with frequent gene mutations including SF3B1 (20-30\%), TET2 ( 20\%), ASXL1 $(\sim 14-15 \%)$, and RUNX1 $(\sim 8-9 \%)^{5-7}$. We pursued this study to assess the impact of degree of bone marrow dysplasia, in the context of gene mutations in patients with WHO defined MDS-RS.

Correspondence: Mrinal M. Patnaik (patnaik.mrinal@mayo.edu)
'Department of Hematology, Mayo Clinic, Rochester, USA

${ }^{2}$ Department of Pathology, Mayo Clinic, Rochester, USA
Successive cases of MDS-RS, meeting the 2016 WHO criteria were identified from our institutional database from years 1994 to 2015. All patients had BM biopsies \& cytogenetics at diagnosis. BM slides including Prussian blue stains for RS were re-reviewed to ensure compliance with the WHO criteria. Targeted exome sequencing for the following genes; TET2, ASXL1, DNMT3A, IDH1, IDH2, TP53, SRSF2, SF3B1, SH2B3, NPM1, FLT3, U2AF1, ZRSR2, JAK2, CSF3R, MPL, MFSD11, CEBPA, SETBP1, ZRSR2, RUNX1, IKZF1, CALR, KRAS, NRAS, CBL, PTPN11, STAG2, BCOR, and GATA2, was performed on diagnostic BM specimens from 64 patients by previously described methods ${ }^{8}$. Detailed clinical, laboratory and treatment data was collected. Mann-Whitney and Fisher exact test was used to assess differences among numerical and categorical variables between the SLD and MLD groups respectively. Multivariate analysis was performed using the Cox Proportional Hazards model. Statistics were performed via JMP Pro software (version 10).

Seventy six patients with MDS-RS met the study criteria, median age 73 years (range: 44-88), 50 (66\%) males. Of these 57 (75\%) were categorized as MDS-RS-SLD and 19 (25\%) as MDS-RS-MLD. Six (8\%) patients had an abnormal karyotype, which were further stratified into R-IPSS one (1.32\%) intermediate, four (5.26\%) poor, and one (1.32\%) very poor cytogenetic risk groups. Overall R-IPSS stratification included 2 (2\%) very low, 40 (53\%) low, 22 (29\%) intermediate, 9 (12\%) high, and $3(4 \%)$ very high-risk categories respectively. Targeted exome sequencing was available in 64 cases and demonstrated the following mutational frequencies; SF3B1 77\%, ASXL1 16\%, DNMT3A 
Table 1 Table displaying distribution of variables between MDS-RS, MDS-RS-SLD, and MDS-RS-MLD

\begin{tabular}{|c|c|c|c|c|}
\hline $\begin{array}{l}\text { Variable; median value } \\
\text { (range or \%) }\end{array}$ & $\begin{array}{l}\text { MDS-RS } \\
(n=76)\end{array}$ & $\begin{array}{l}\text { SLD } \\
(n=57)\end{array}$ & $\begin{array}{l}\text { MLD } \\
(n=19)\end{array}$ & $\begin{array}{l}P \text { - } \\
\text { value }\end{array}$ \\
\hline Age (years) & $73(44-88)$ & $73(47-88)$ & $72(44-81)$ & 0.22 \\
\hline No. of males & $50(66)$ & $36(63)$ & $14(74)$ & 0.4 \\
\hline $\mathrm{Hb} ; \mathrm{gm} / \mathrm{dl}$ & $\begin{array}{l}9.3 \\
(5.8-14.4)\end{array}$ & $\begin{array}{l}9.3 \\
(5.8-13.4)\end{array}$ & $\begin{array}{l}10 \\
(7.8-14.4)\end{array}$ & 0.17 \\
\hline WBC count $\times 10^{9}$ per liter & $\begin{array}{l}5.1 \\
(1.2-17.6)\end{array}$ & $\begin{array}{l}5.7 \\
(2.2-17.6)\end{array}$ & $\begin{array}{l}4.4 \\
(1.2-9.4)\end{array}$ & $0.02 *$ \\
\hline ANC $\times 10^{9}$ per liter & $\begin{array}{l}3.06 \\
(0.3-12.5)\end{array}$ & $\begin{array}{l}3.2 \\
(0.6-12.5)\end{array}$ & $\begin{array}{l}2.13 \\
(0.3-6.3)\end{array}$ & $0.02 *$ \\
\hline Platelet count $\times 10^{9}$ per liter & $\begin{array}{l}237 \\
(7-819)\end{array}$ & $\begin{array}{l}255 \\
(7-819)\end{array}$ & $\begin{array}{l}202 \\
(27-438)\end{array}$ & $0.05 *$ \\
\hline $\mathrm{BM}$ ringed sideroblasts & $35(10-80)$ & $50(10-80)$ & $20(15-70)$ & 0.004 * \\
\hline \multicolumn{4}{|l|}{ Cytogenetics } & 0.6 \\
\hline $\begin{array}{l}\text { Abnormal karyotype (R-IPSS } \\
\text { intermediate, poor and very } \\
\text { poor cytogenetic risk } \\
\text { groups) } \\
\text { IPSS cytogenetic risk groups }\end{array}$ & 6 (8) & $4(8)$ & $2(11)$ & 0.5 \\
\hline Good & $69(91)$ & $52(91)$ & $17(11)$ & 0.5 \\
\hline Intermediate & $2(3)$ & $2(4)$ & $0(0)$ & \\
\hline Poor & $5(6)$ & $3(5)$ & $2(11)$ & \\
\hline \multicolumn{5}{|l|}{ R-IPSS cytogenetic risk groups } \\
\hline Very good & $2(3)$ & $1(2)$ & $1(5)$ & 0.6 \\
\hline Good & $67(88)$ & $51(89)$ & $16(84)$ & \\
\hline Intermediate & $2(3)$ & $2(4)$ & $0(0)$ & \\
\hline Poor & $4(5)$ & $2(4)$ & $2(11)$ & \\
\hline Very poor & $1(1)$ & $1(2)$ & $0(0)$ & \\
\hline \multicolumn{5}{|l|}{ Genomic abnormalities } \\
\hline SF3B1 & $49(77)$ & $41(82)$ & $8(57)$ & 0.06 \\
\hline$A S X L 1$ & $10(16)$ & $6(12)$ & $4(29)$ & 0.15 \\
\hline DNMT3A & $8(13)$ & $8(16)$ & $0(0)$ & $0.04 *$ \\
\hline TET2 & $4(6)$ & $4(8)$ & $0(0)$ & 0.2 \\
\hline TP53 & $3(5)$ & $3(6)$ & $0(0)$ & 0.2 \\
\hline $\mid \mathrm{DH} 1$ & $2(3)$ & $2(4)$ & $0(0)$ & 0.3 \\
\hline SRSF2 & $1(2)$ & $1(2)$ & $0(0)$ & - \\
\hline $\begin{array}{l}\text { Others (PTPN11, ZRSR2, } \\
\text { CSF3R, U2AF1) }\end{array}$ & $4(6)$ & $4(8)$ & $0(0)$ & - \\
\hline \multicolumn{5}{|l|}{ Treatment } \\
\hline HMA treatment & $5(7)$ & $2(4)$ & $3(16)$ & 0.2 \\
\hline
\end{tabular}

Table 1 continued

\begin{tabular}{lllll}
\hline $\begin{array}{l}\text { Variable; median value } \\
\text { (range or \%) }\end{array}$ & $\begin{array}{l}\text { MDS-RS } \\
(\boldsymbol{n}=\mathbf{7 6})\end{array}$ & $\begin{array}{l}\text { SLD } \\
(\boldsymbol{n}=\mathbf{5 7})\end{array}$ & $\begin{array}{l}\text { MLD } \\
(\boldsymbol{n}=\mathbf{1 9})\end{array}$ & $\begin{array}{l}\boldsymbol{P} \text { - } \\
\text { value }\end{array}$ \\
\hline $\begin{array}{l}\text { Immunomodulatory } \\
\text { treatment (Lenalidomide) }\end{array}$ & $1(1)$ & $1(2)$ & $0(0)$ & 0.83 \\
$\begin{array}{l}\text { Allogeneic HSCT } \\
\begin{array}{l}\text { Outcomes } \\
\text { Leukemic transformation }\end{array}\end{array}$ & $2(3)$ & $1(2)$ & $1(5)$ & 0.16 \\
$\begin{array}{l}\text { Overall survival; median } \\
\text { months (range) }\end{array}$ & $46(0-190)$ & $47(0-190)$ & $44(6-123)$ & 0.2 \\
\hline & & & & \\
\hline * Signify statistically significant values & & & \\
\end{tabular}

13\%, TET2 6\%, TP53 5\%, IDH1 3, and 2\% each for SRSF2, PTPN11, ZRSR2, CSF3R, and U2AF1 (see Table 1).

In comparison to MDS-RS-MLD, patients with MDSRS-SLD had a higher frequency of $S F 3 B 1$ (82 vs $57 \%, p=$ 0.06 ) and DNMT3A (16 vs $0 \%, p=0.04)$ mutations, however the frequency of $A S X L 1$ (12 vs $29 \%, p=0.15$ ) mutations was lower. At a median follow-up of 33 months, 68 (89\%) deaths and $2(3 \%)$ leukemic transformations were documented. Median survival (OS) of the entire cohort was 46 months (Range: 0-190 months). Survival for the MDS-RS-SLD group was 47 months, while that for MDS-RS-MLD was 44 months [Hazard ratio (HR) 1.4, $p=0.2$ ]. On a univariate survival analysis that included age, sex, hemoglobin, white blood cell count with individual differential counts, platelet count, BM morphology (SLD versus MLD), peripheral blood blasts, cytogenetics and the aforementioned gene mutations, lack of SF3B1 mutations (HR 4.35, 95\% CI 2.2-8.3, $p<0.0001$, Fig. 1a) and presence of ASXL1 mutation (HR 3.13, 95\% CI 1.4-6.3, $p=0.006$, Fig. 1b) adversely impacted OS. Interestingly, multilineage dysplasia did not show a statistically significant correlation with outcomes (HR 1.4, 95\% CI 0.8-2.5, $p=0.2$ ) (Fig. 1c). In a multivariate analysis that included $A S X L 1$ and SF3B1 mutations as variables, both the presence of $A S X L 1$ mutations [HR of 2.3 (95\% CI 1.0-4.7), $p=0.05]$ and the absence of SF3B1 mutations [HR 3.7 (95\% CI 1.8-7.2), $p=0.0006$ ] retained an independent and negative prognostic impact. In addition, both these mutations retained their prognostic impact when analyzed in the context of R-IPSS risk categories. Further, we assessed the combined effect of presence (mutated or $\mathrm{mt}$ ) or absence (wild-type or wt) of $A S X L 1$ and SF3B1 mutations on outcomes. Median OS was longest in the $A S X L 1 \mathrm{wt} / S F 3 B 1 \mathrm{mt} \quad(n=42$, 69 months) sub-group of patients in comparison to others $(p<0.0001)$, suggesting that the presence of both these mutations need to be assessed at diagnosis for an accurate prognostication (Fig. 1d). Patient numbers were limited for individual comparison with other sub-groups. 

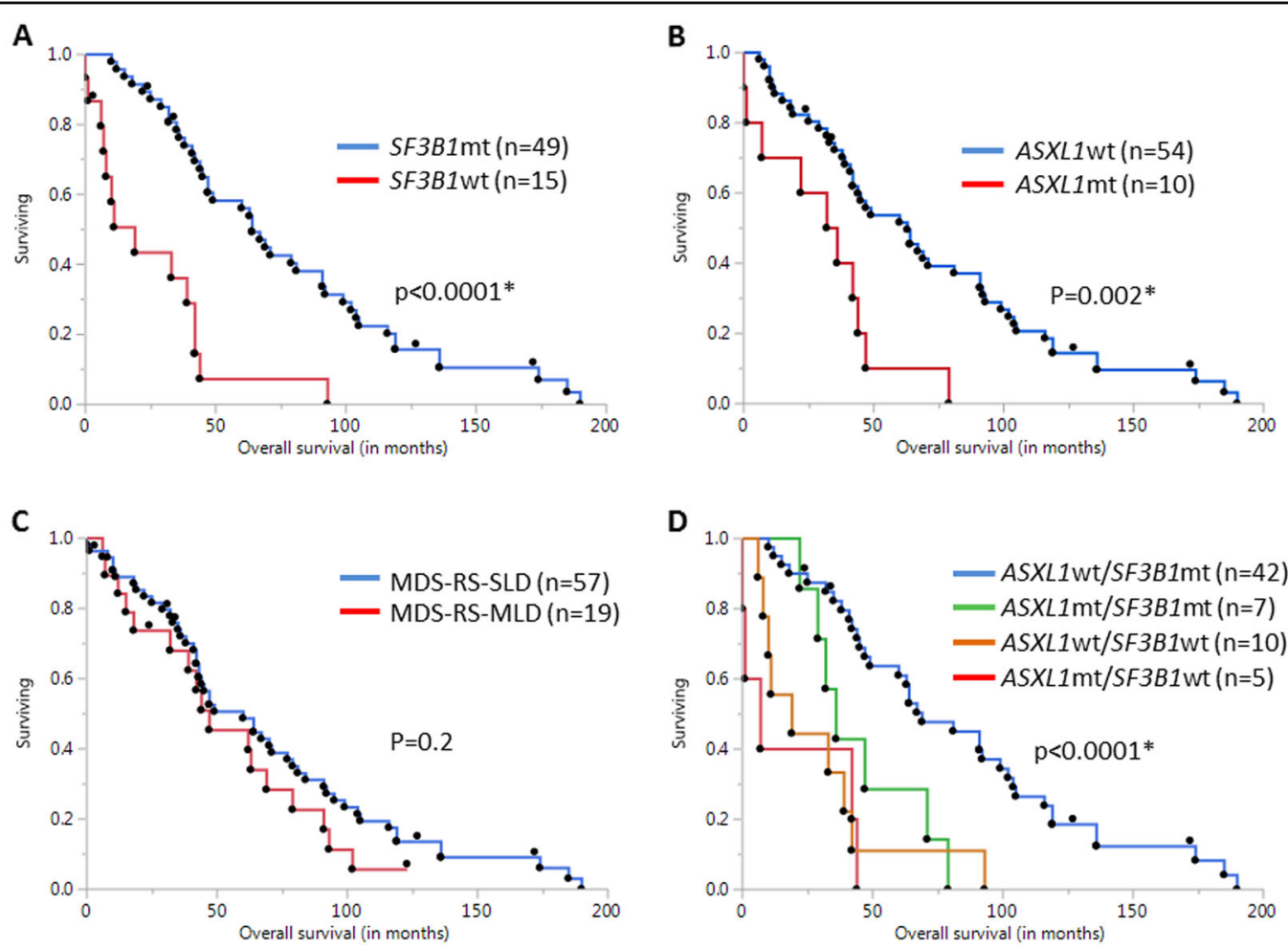

Fig. 1 Kaplan-Meir survival analysis for MDS-RS patients based on somatic mutational analysis. a shows Kaplan-Meir survival curves for $S F 3 B 1$ mutated $(\mathrm{mt})$ and wild-type $(\mathrm{wt})$ patients. Log-rank test was used to assess difference between groups. Median OS is significantly higher in the SF3B1 mt patients (64 versus 19 months, $p<0.0001^{*}$ ). $\mathbf{b}$ shows a similar analysis, depending on ASXL1 mutational status. Median OS in ASXL1 wt patients is much higher than ASXL1 mt patients (64 versus 39 months, $p=0.002^{*}$ ). c shows Kaplan-Meir survival curves among the two sub-groups based on bone marrow morphology (MDS-RS-SLD and MDS-RS-MLD). No significant survival difference was found between the two groups $(p=0.2)$. $\mathbf{d}$ shows Kaplan-Meir survival curves for four sub-groups, based on presence or absence of both SF3B1 and ASXL1 mutations. Median OS was highest in ASXL1 wt/SF3B1 mt ( $n=42,69$ months) sub-group ( $\left.p<0.0001^{*}\right)$, followed by ASXL1 mt/SF3B1 mt ( $n=7,39$ months), ASXL1 wt/SF3B1 wt ( $\left.n=10,19 \mathrm{months}\right)$ and ASXL1 mt/SF3B1 wt ( $n=5,7$ months) sub-groups, respectively

Incorporation of genomic alterations into existing WHO morphological classifications is critical for better classifying these disorders and to refine existing prognostic strategies 9 . Our study validates the prognostic impact of gene mutations in MDS-RS and also assesses their relevance in the context of existing morphological distinctions.

Mutations in SF3B1, a gene regulating pre-mRNA splicing, are known to have favorable outcomes in MDS-RS ${ }^{3,10}$ and our study has confirmed this observation. In contrast, $A S X L 1$ mutations impact chromatin regulation by impairing activity of polycomb repressive complex 2 (PRC2), and are associated with adverse outcomes in myeloid neoplasms such as MDS, chronic myelomonocytic leukemia and myelofibrosis ${ }^{8,11,12}$. To our knowledge, ours is the first study to assess the prognostic impact of ASXL1 mutations in an independent large cohort of WHO-defined MDS-RS patients and has demonstrated an adverse impact on survival. In particular, the subgroup of MDS-RS patients with mutated SF3B1 and wild-type ASXL1 has the best prognosis. In conclusion, molecular abnormalities involving relevant genes take precedence in terms of prognostication within morphologically defined subsets of MDS and in fact, may be more relevant than existing morphological prognosticators such as degree of bone marrow dysplasia. Collaborative prospective efforts with larger number of patients are needed to validate these findings.

\section{Acknowledgements}

Current publication is supported in part by grants from the "The Gerstner Family Career Development Award" and the Mayo Clinic Center for Individualized Medicine, Mayo Clinic, Rochester, MN, USA". This publication was supported by CTSA Grant Number KL2 TR000136 from the National Center for Advancing Translational Science (NCATS). Its contents are solely the responsibility of the authors and do not necessarily represent the official views of the $\mathrm{NIH}$.

\section{Conflict of interest}

The authors declare that they have no conflict of interest.

\section{Publisher's note}

Springer Nature remains neutral with regard to jurisdictional claims in published maps and institutional affiliations. 
Received: 15 December 2017 Accepted: 2 January 2018

Published online: 12 February 2018

\section{References}

1. Arber, D. A. et al. The2016 revision to the World Health Organization classification of myeloid neoplasms and acute leukemia. Blood 127, 2391-2405 (2016).

2. Vardiman, J. W. et al. The2008 revision of the World Health Organization (WHO) classification of myeloid neoplasms and acute leukemia: rationale and important changes. Blood 114, 937-951 (2009).

3. Patnaik, M. M. et al. SF3B1 mutations are prevalent in myelodysplastic syndromes with ring sideroblasts but do not hold independent prognostic value. Blood 119, 569-572 (2012).

4. Patnaik, M. M. et al. Prognostic irrelevance of ring sideroblast percentage in World Health Organization-defined myelodysplastic syndromes without excess blasts. Blood 119, 5674-5677 (2012).

5. Bejar, R. et al. Clinical effect of point mutations in myelodysplastic syndromes. N. Engl. J. Med. 364, 2496-2506 (2011).
6. Papaemmanuil, E. et al. Somatic SF3B1 mutation in myelodysplasia with ring sideroblasts. N. Engl. J. Med. 365, 1384-1395 (2011).

7. Patnaik, M. M. \& Tefferi, A. Refractory anemia with ring sideroblasts (RARS) and RARS with thrombocytosis (RARS-T): 2017 update on diagnosis, risk-stratification, and management. Am. J. Hematol. 92, 297-310 (2017).

8. Patnaik, M. M. et al. Prognostic interaction between ASXL1 and TET2 mutations in chronic myelomonocytic leukemia. Blood. Cancer J. 6, e385 (2016).

9. Tefferi, A. et al. Targeted next-generation sequencing in myelodysplastic syndromes and prognostic interaction between mutations and IPSS-R. Am. J. Hematol. 92, 1311-1317 (2017).

10. Malcovati, L. et al. SF3B1 mutation identifies a distinct subset of myelodysplastic syndrome with ring sideroblasts. Blood 126, 233-241 (2015).

11. Bejar, R. et al. Validation of a prognostic model and the impact of mutations in patients with lower-risk myelodysplastic syndromes. J. Clin. Oncol. 30, 3376-3382 (2012)

12. Thol, F. et al. Prognostic significance of ASXL1 mutations in patients with myelodysplastic syndromes. J. Clin. Oncol. 29, 2499-2506 (2011). 